Acta Crystallographica Section E

Structure Reports

Online

ISSN 1600-5368

\section{Basavegowda Nagaraj, ${ }^{\text {a }}$ Hemmige S. Yathirajan, Padmarajaiah Nagaraja ${ }^{a}$ and Daniel E. Lynch ${ }^{\mathbf{b}}$.}

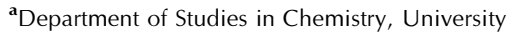
of Mysore, Manasagangotri, Mysore 570 006, India, and ${ }^{\mathbf{b}}$ School of Science and the Environment, Coventry University, Coventry CV1 5FB, UK

Correspondence e-mail: apx106@coventry.ac.uk

\section{Key indicators}

Single-crystal X-ray study

$T=120 \mathrm{~K}$

Mean $\sigma(\mathrm{C}-\mathrm{C})=0.002 \AA$

$R$ factor $=0.038$

$w R$ factor $=0.103$

Data-to-parameter ratio $=15.1$

For details of how these key indicators were automatically derived from the article, see http://journals.iucr.org/e.

\title{
1-(Carbamoylmethyl)cyclohexanecarboxylic acid
}

Molecules of the title compound, $\mathrm{C}_{9} \mathrm{H}_{15} \mathrm{NO}_{3}$, form a twodimensional hydrogen-bonded network, via $\mathrm{O}-\mathrm{H} \cdots \mathrm{O}$ and $\mathrm{N}-\mathrm{H} \cdots \mathrm{O}$ interactions, which runs parallel to the $b c$ plane. In this structure, neither the carboxylic acid groups nor the carbamoyl groups are involved in dimer formations.

\section{Comment}

The title compound, (I), is used as an intermediate in the synthesis of biologically active heterocycles (LaRoche \& Helmers, 2004). A search of the Cambridge Structural Database (Version 5.26; Allen, 2002) reveals that there are 11 structures of 1,1-disubstituted cyclohexane with a carboxylic acid group as one of the substituents. Of these, only three contain 1-cyclohexanecarboxylic acid itself. The remaining structures each contain an amino group (as the second substituent), with further attached groups on the amino $\mathrm{N}$ atom. There are no structures similar to 1-(carbamoylmethyl)cyclohexane.

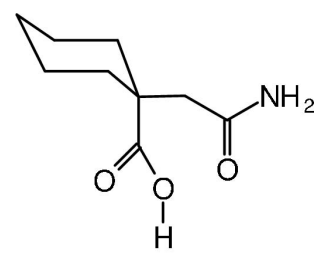

(I)

Molecules of the title compound (Fig. 1) form a twodimensional hydrogen-bonded network, via $\mathrm{O}-\mathrm{H} \cdots \mathrm{O}$ and $\mathrm{N}-\mathrm{H} \cdots \mathrm{O}$ interactions, which runs parallel to the $b c$ plane. Hydrogen-bonding associations are listed in Table 1. The carboxylic $\mathrm{OH}$ group hydrogen bonds to the carbamoyl $\mathrm{O}$ atom of an adjacent molecule while the amino group of that molecule, in return, hydrogen bonds with the carbamoyl $\mathrm{O}$ atom of the first molecule. These two associations form a hydrogen-bonded ring motif [ $R_{2}^{2}(11)$ graph set (Etter, 1990)] that, when repeated, propagates the hydrogen-bonding network in the $b$-axis direction. An $\mathrm{N}-\mathrm{H} \cdots \mathrm{O}$ association between the second amino $\mathrm{H}$ atom and an adjacent carboxyl carbonyl $\mathrm{O}$ atom in the $c$-axis direction generates the twodimensional network. Interestingly, in this structure, neither the carboxylic acid groups nor the carbamoyl groups are involved in $R_{2}^{2}(8)$ graph-set dimer formations, with like groups or with each other.

\section{Experimental}

Cyclohexanone $(1.04 \mathrm{~g}, 10 \mathrm{mmol})$ was treated with ethyl cyanoacetate $(1.06 \mathrm{~g}, 10 \mathrm{mmol})$ in the presence of $\mathrm{NaOH}(5 \mathrm{ml}, 10 \%$
Received 22 February 2005 Accepted 25 February 2005 Online 11 March 2005
(C) 2005 International Union of Crystallography Printed in Great Britain - all rights reserved 
aqueous solution). The resultant compound was further treated with $\mathrm{NaCN}(0.49 \mathrm{~g}, 10 \mathrm{mmol})$ in ethanol $(5 \mathrm{ml})$, and hydrolysed to obtain the title compound. Crystals were grown from methanol.

$$
\begin{aligned}
& \text { Crystal data } \\
& \mathrm{C}_{9} \mathrm{H}_{15} \mathrm{NO}_{3} \\
& M_{r}=185.22 \\
& \text { Monoclinic, } P 2_{1} / c \\
& a=13.4973(5) \AA \\
& b=8.0905(2) \AA \\
& c=8.8358(3) \AA \\
& \beta=102.627(2)^{\circ} \\
& V=941.53(5) \AA^{3} \\
& Z=4
\end{aligned}
$$

$$
\begin{aligned}
& D_{x}=1.307 \mathrm{Mg} \mathrm{m}^{-3} \\
& \text { Mo } K \alpha \text { radiation } \\
& \text { Cell parameters from } 2268 \\
& \quad \text { reflections } \\
& \theta=2.9-27.5^{\circ} \\
& \mu=0.10 \mathrm{~mm}^{-1} \\
& T=120(2) \mathrm{K} \\
& \text { Prism, colourless } \\
& 0.65 \times 0.30 \times 0.10 \mathrm{~mm}
\end{aligned}
$$

\section{Data collection}

Nonius KappaCCD diffractometer $\varphi$ and $\omega$ scans

Absorption correction: multi-scan (SADABS; Sheldrick, 2003)

$T_{\min }=0.939, T_{\max }=0.990$

10959 measured reflections

1842 independent reflections

\section{Refinement}

Refinement on $F^{2}$

$R\left[F^{2}>2 \sigma\left(F^{2}\right)\right]=0.038$

$w R\left(F^{2}\right)=0.103$

$S=1.05$

1842 reflections

122 parameters

$\mathrm{H}$ atoms treated by a mixture of independent and constrained refinement

$$
\begin{aligned}
& 1627 \text { reflections with } I>2 \sigma(I) \\
& R_{\text {int }}=0.030 \\
& \theta_{\max }=26.0^{\circ} \\
& h=-16 \rightarrow 16 \\
& k=-9 \rightarrow 9 \\
& l=-10 \rightarrow 10
\end{aligned}
$$

$$
\begin{aligned}
& w=1 /\left[\sigma^{2}\left(F_{o}^{2}\right)+(0.0503 P)^{2}\right. \\
& +0.4339 P] \\
& \text { where } P=\left(F_{o}^{2}+2 F_{c}{ }^{2}\right) / 3 \\
& (\Delta / \sigma)_{\max }=0.001 \\
& \Delta \rho_{\max }=0.20 \mathrm{e}^{-3} \\
& \Delta \rho_{\min }=-0.25 \mathrm{e}^{-3}
\end{aligned}
$$

\section{Table 1}

Hydrogen-bonding geometry $\left(\AA,^{\circ}\right)$.

\begin{tabular}{lllll}
\hline$D-\mathrm{H} \cdots A$ & $D-\mathrm{H}$ & $\mathrm{H} \cdots A$ & $D \cdots A$ & $D-\mathrm{H} \cdots A$ \\
\hline $\mathrm{O} 3-\mathrm{H} 3 \cdots \mathrm{O} 1^{\mathrm{i}}$ & $0.963(18)$ & $1.640(19)$ & $2.5829(13)$ & $165(2)$ \\
$\mathrm{N} 1-\mathrm{H} 1 \cdots \mathrm{O} 1^{\mathrm{ii}}$ & 0.88 & 2.21 & $3.0680(15)$ & 164 \\
$\mathrm{~N} 1-\mathrm{H} 2 \cdots \mathrm{O} 2^{\mathrm{iii}}$ & 0.88 & 2.12 & $2.9635(15)$ & 162 \\
\hline
\end{tabular}

Symmetry codes: (i) $-x, y-\frac{1}{2}, \frac{1}{2}-z$; (ii) $-x, \frac{1}{2}+y, \frac{1}{2}-z$; (iii) $x, \frac{1}{2}-y, z-\frac{1}{2}$.

The carboxyl $\mathrm{H}$ atom was located in a difference Fourier synthesis and its positional parameters were refined. Other $\mathrm{H}$ atoms were included in the refinement at calculated positions, in the riding-model approximation, with $\mathrm{C}-\mathrm{H}$ distances of $0.99 \AA$ and $\mathrm{N}-\mathrm{H}$ distances of $0.88 \AA$. The isotropic displacement parameters for all $\mathrm{H}$ atoms were set equal to $1.25 U_{\text {eq }}$ of the carrier atom.

Data collection: COLLECT (Hooft, 1998); cell refinement: DENZO (Otwinowski \& Minor, 1997) and COLLECT; data reduc-

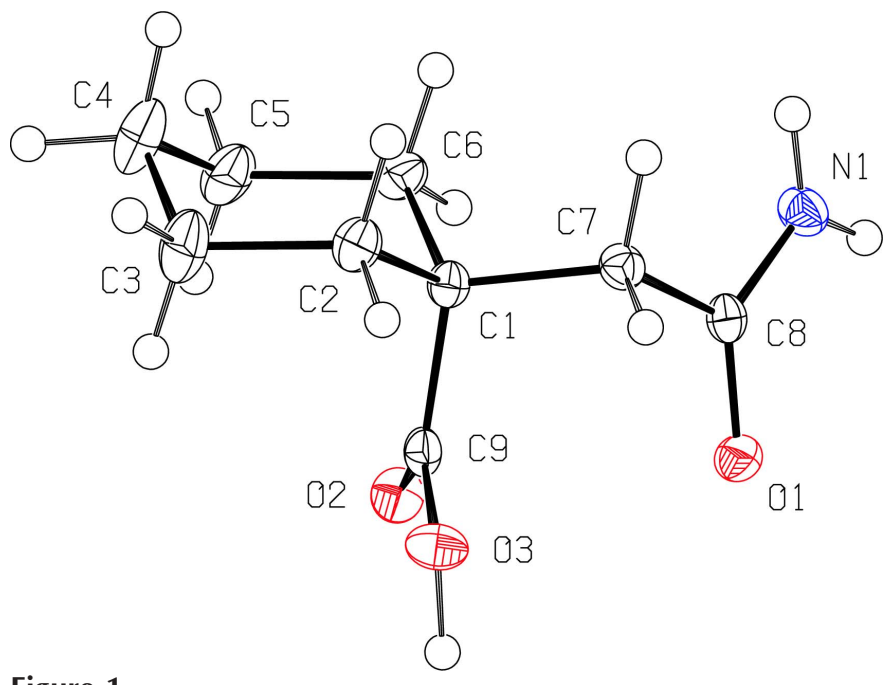

Figure 1

The molecular configuration and atom-numbering scheme for (I). Displacement ellipsoids are drawn at the $50 \%$ probability level and $\mathrm{H}$ atoms are drawn as spheres of arbitrary radius.

tion: DENZO and COLLECT; program(s) used to solve structure: SHELXS97 (Sheldrick, 1997); program(s) used to refine structure: SHELXL97 (Sheldrick, 1997); molecular graphics: PLATON (Spek, 2003); software used to prepare material for publication: SHELXL97.

The authors thank the EPSRC National Crystallography Service (Southampton, England) and acknowledge the use of the EPSRC's Chemical Database Service at Daresbury (Fletcher et al., 1996).

\section{References}

Allen, F. H. (2002). Acta Cryst. B58, 380-388.

Etter, M. C. (1990). Acc. Chem. Res. 23, 120-126.

Fletcher, D. A., McMeeking, R. F. \& Parkin, D. J. (1996). J. Chem. Inf. Comput. Sci. 36, 746-749.

Hooft, R. W. W. (1998). COLLECT. Nonius BV, Delft, The Netherlands.

LaRoche, S. M. \& Helmers, S. L. (2004). JAMA, 291, 605-614.

Otwinowski, Z. \& Minor, W. (1997). Methods in Enzymology, Vol. 276, Macromolecular Crystallography, Part A, edited by C. W. Carter Jr and R. M. Sweet, pp. 307-326. New York: Academic Press.

Sheldrick, G. M. (1997). SHELXS97 and SHELXL97. University of Göttingen, Germany.

Sheldrick, G. M. (2003). SADABS. Bruker AXS Inc., Madison, Wisconsin, USA.

Spek, A. L. (2003). J. Appl. Cryst. 36, 7-13. 


\section{supporting information}

Acta Cryst. (2005). E61, o884-o885 [https://doi.org/10.1107/S1600536805006148]

\section{1-(Carbamoylmethyl)cyclohexanecarboxylic acid}

Basavegowda Nagaraj, Hemmige S. Yathirajan, Padmarajaiah Nagaraja and Daniel E. Lynch

1-(Carbamoylmethyl)cyclohexanecarboxylic acid

Crystal data

$\mathrm{C}_{9} \mathrm{H}_{15} \mathrm{NO}_{3}$

$M_{r}=185.22$

Monoclinic, $P 2_{1} / c$

Hall symbol: $-\mathrm{P} 2 \mathrm{ybc}$

$a=13.4973(5) \AA$

$b=8.0905(2) \AA$

$c=8.8358(3) \AA$

$\beta=102.627(2)^{\circ}$

$V=941.53(5) \AA^{3}$

$Z=4$

$F(000)=400$

$D_{\mathrm{x}}=1.307 \mathrm{Mg} \mathrm{m}^{-3}$

Melting point: $437 \mathrm{~K}$

Mo $K \alpha$ radiation, $\lambda=0.71073 \AA$

Cell parameters from 2268 reflections

$\theta=2.9-27.5^{\circ}$

$\mu=0.10 \mathrm{~mm}^{-1}$

$T=120 \mathrm{~K}$

Prism, colourless

$0.65 \times 0.30 \times 0.10 \mathrm{~mm}$

Data collection

Nonius KappaCCD diffractometer

Radiation source: Bruker Nonius FR591 rotating anode

$10 \mathrm{~cm}$ confocal mirrors monochromator

Detector resolution: 9.091 pixels $\mathrm{mm}^{-1}$

$\varphi$ and $\omega$ scans

Absorption correction: multi-scan

(SADABS; Sheldrick, 2003)

$T_{\min }=0.939, T_{\max }=0.990$

10959 measured reflections

1842 independent reflections

1627 reflections with $I>2 \sigma(I)$

$R_{\text {int }}=0.030$

$\theta_{\max }=26.0^{\circ}, \theta_{\min }=3.5^{\circ}$

$h=-16 \rightarrow 16$

$k=-9 \rightarrow 9$

$l=-10 \rightarrow 10$

\section{Refinement}

Refinement on $F^{2}$

Least-squares matrix: full

$R\left[F^{2}>2 \sigma\left(F^{2}\right)\right]=0.038$

$w R\left(F^{2}\right)=0.103$

$S=1.05$

1842 reflections

122 parameters

0 restraints

Primary atom site location: structure-invariant direct methods

Secondary atom site location: difference Fourier map

Hydrogen site location: inferred from neighbouring sites

$\mathrm{H}$ atoms treated by a mixture of independent and constrained refinement

$w=1 /\left[\sigma^{2}\left(F_{\mathrm{o}}^{2}\right)+(0.0503 P)^{2}+0.4339 P\right]$ where $P=\left(F_{\mathrm{o}}^{2}+2 F_{\mathrm{c}}^{2}\right) / 3$

$(\Delta / \sigma)_{\max }=0.001$

$\Delta \rho_{\max }=0.20 \mathrm{e} \AA^{-3}$

$\Delta \rho_{\min }=-0.25$ e $\AA^{-3}$

Extinction correction: SHELXL97, $\mathrm{Fc}^{*}=\mathrm{kFc}\left[1+0.001 \times \mathrm{Fc}^{2} \lambda^{3} / \sin (2 \theta)\right]^{-1 / 4}$

Extinction coefficient: 0.048 (6)

Fractional atomic coordinates and isotropic or equivalent isotropic displacement parameters $\left(\AA^{2}\right)$

\begin{tabular}{lllll}
\hline & $x$ & $y$ & $z$ & $U_{\text {iso }} * / U_{\text {eq }}$ \\
\hline O1 & $0.01177(7)$ & $0.16623(11)$ & $0.19598(11)$ & $0.0192(3)$
\end{tabular}




$\begin{array}{lllll}\text { O2 } & 0.18173(7) & -0.03683(12) & 0.38790(11) & 0.0214(3) \\ \text { O3 } & 0.11848(8) & -0.18613(12) & 0.17583(11) & 0.0224(3) \\ \text { H3 } & 0.0775(13) & -0.237(2) & 0.239(2) & 0.028^{*} \\ \text { N1 } & 0.07782(9) & 0.40190(14) & 0.12383(13) & 0.0202(3) \\ \text { H1 } & 0.0420 & 0.4629 & 0.1748 & 0.025^{*} \\ \text { H2 } & 0.1191 & 0.4493 & 0.0724 & 0.025^{*} \\ \text { C1 } & 0.22107(9) & 0.04376(16) & 0.14274(15) & 0.0157(3) \\ \text { C2 } & 0.27157(10) & -0.06508(17) & 0.03825(16) & 0.0207(3) \\ \text { H21 } & 0.2217 & -0.1471 & -0.0157 & 0.026^{*} \\ \text { H22 } & 0.2921 & 0.0050 & -0.0415 & 0.026^{*} \\ \text { C3 } & 0.36471(11) & -0.1557(2) & 0.1304(2) & 0.0310(4) \\ \text { H31 } & 0.3966 & -0.2201 & 0.0585 & 0.039^{*} \\ \text { H32 } & 0.3434 & -0.2340 & 0.2033 & 0.039^{*} \\ \text { C4 } & 0.44223(11) & -0.0342(2) & 0.2213(2) & 0.0354(4) \\ \text { H41 } & 0.4999 & -0.0963 & 0.2844 & 0.044^{*} \\ \text { H42 } & 0.4688 & 0.0368 & 0.1480 & 0.044^{*} \\ \text { C5 } & 0.39417(11) & 0.0735(2) & 0.32693(18) & 0.0271(4) \\ \text { H51 } & 0.4443 & 0.1561 & 0.3789 & 0.034^{*} \\ \text { H52 } & 0.3751 & 0.0037 & 0.4081 & 0.034^{*} \\ \text { C6 } & 0.29991(10) & 0.16275(17) & 0.23608(16) & 0.0199(3) \\ \text { H61 } & 0.3207 & 0.2429 & 0.1642 & 0.025^{*} \\ \text { H62 } & 0.2683 & 0.2255 & 0.3094 & 0.025^{*} \\ \text { C7 } & 0.13317(10) & 0.14047(17) & 0.03674(15) & 0.0169(3) \\ \text { H71 } & 0.1622 & 0.2166 & -0.0298 & 0.021^{*} \\ \text { H72 } & 0.0889 & 0.0611 & -0.0320 & 0.021^{*} \\ \text { C8 } & 0.06951(9) & 0.23871(17) & 0.12452(14) & 0.0159(3) \\ \text { C9 } & 0.17348(10) & -0.06333(16) & 0.25029(15) & 0.0161(3) \\ & & & & \end{array}$

Atomic displacement parameters $\left(\AA^{2}\right)$

\begin{tabular}{lllllll}
\hline & $U^{11}$ & $U^{22}$ & $U^{33}$ & $U^{12}$ & $U^{13}$ & $U^{23}$ \\
\hline O1 & $0.0178(5)$ & $0.0183(5)$ & $0.0229(5)$ & $0.0012(4)$ & $0.0078(4)$ & $0.0003(4)$ \\
O2 & $0.0227(5)$ & $0.0249(5)$ & $0.0166(5)$ & $0.0010(4)$ & $0.0041(4)$ & $-0.0012(4)$ \\
O3 & $0.0279(6)$ & $0.0187(5)$ & $0.0218(5)$ & $-0.0066(4)$ & $0.0077(4)$ & $-0.0022(4)$ \\
N1 & $0.0241(6)$ & $0.0163(6)$ & $0.0219(6)$ & $0.0026(5)$ & $0.0088(5)$ & $0.0015(5)$ \\
C1 & $0.0153(6)$ & $0.0155(6)$ & $0.0161(7)$ & $0.0014(5)$ & $0.0031(5)$ & $-0.0009(5)$ \\
C2 & $0.0200(7)$ & $0.0217(7)$ & $0.0216(7)$ & $0.0030(5)$ & $0.0069(6)$ & $-0.0030(6)$ \\
C3 & $0.0230(8)$ & $0.0316(9)$ & $0.0376(9)$ & $0.0101(6)$ & $0.0051(7)$ & $-0.0063(7)$ \\
C4 & $0.0167(7)$ & $0.0444(10)$ & $0.0433(10)$ & $0.0075(7)$ & $0.0024(7)$ & $-0.0053(8)$ \\
C5 & $0.0166(7)$ & $0.0317(8)$ & $0.0302(8)$ & $-0.0026(6)$ & $-0.0009(6)$ & $-0.0044(6)$ \\
C6 & $0.0174(7)$ & $0.0201(7)$ & $0.0224(7)$ & $-0.0027(5)$ & $0.0046(6)$ & $-0.0025(5)$ \\
C7 & $0.0184(7)$ & $0.0176(7)$ & $0.0151(6)$ & $0.0011(5)$ & $0.0042(5)$ & $0.0013(5)$ \\
C8 & $0.0151(6)$ & $0.0181(7)$ & $0.0126(6)$ & $0.0015(5)$ & $-0.0009(5)$ & $0.0010(5)$ \\
C9 & $0.0145(6)$ & $0.0145(6)$ & $0.0187(7)$ & $0.0038(5)$ & $0.0022(5)$ & $0.0003(5)$
\end{tabular}


Geometric parameters $\left(\AA,{ }^{\circ}\right)$

\begin{tabular}{|c|c|c|c|}
\hline $\mathrm{O} 1-\mathrm{C} 8$ & $1.2510(16)$ & $\mathrm{C} 3-\mathrm{C} 4$ & $1.529(2)$ \\
\hline $\mathrm{O} 2-\mathrm{C} 9$ & $1.2156(16)$ & $\mathrm{C} 3-\mathrm{H} 31$ & 0.99 \\
\hline $\mathrm{O} 3-\mathrm{C} 9$ & $1.3257(16)$ & $\mathrm{C} 3-\mathrm{H} 32$ & 0.99 \\
\hline $\mathrm{O} 3-\mathrm{H} 3$ & $0.963(18)$ & $\mathrm{C} 4-\mathrm{C} 5$ & $1.522(2)$ \\
\hline $\mathrm{N} 1-\mathrm{C} 8$ & $1.3252(18)$ & $\mathrm{C} 4-\mathrm{H} 41$ & 0.99 \\
\hline $\mathrm{N} 1-\mathrm{H} 1$ & 0.88 & $\mathrm{C} 4-\mathrm{H} 42$ & 0.99 \\
\hline $\mathrm{N} 1-\mathrm{H} 2$ & 0.88 & $\mathrm{C} 5-\mathrm{C} 6$ & $1.529(2)$ \\
\hline $\mathrm{C} 1-\mathrm{C} 9$ & $1.5274(18)$ & $\mathrm{C} 5-\mathrm{H} 51$ & 0.99 \\
\hline $\mathrm{C} 1-\mathrm{C} 6$ & $1.5348(18)$ & $\mathrm{C} 5-\mathrm{H} 52$ & 0.99 \\
\hline $\mathrm{C} 1-\mathrm{C} 2$ & $1.5387(18)$ & C6-H61 & 0.99 \\
\hline $\mathrm{C} 1-\mathrm{C} 7$ & $1.5528(17)$ & C6-H62 & 0.99 \\
\hline $\mathrm{C} 2-\mathrm{C} 3$ & $1.528(2)$ & $\mathrm{C} 7-\mathrm{C} 8$ & $1.5043(18)$ \\
\hline $\mathrm{C} 2-\mathrm{H} 21$ & 0.99 & $\mathrm{C} 7-\mathrm{H} 71$ & 0.99 \\
\hline $\mathrm{C} 2-\mathrm{H} 22$ & 0.99 & $\mathrm{C} 7-\mathrm{H} 72$ & 0.99 \\
\hline $\mathrm{C} 9-\mathrm{O} 3-\mathrm{H} 3$ & $111.3(10)$ & $\mathrm{C} 3-\mathrm{C} 4-\mathrm{H} 42$ & 109.5 \\
\hline $\mathrm{C} 8-\mathrm{N} 1-\mathrm{H} 1$ & 120.0 & $\mathrm{H} 41-\mathrm{C} 4-\mathrm{H} 42$ & 108.1 \\
\hline $\mathrm{C} 8-\mathrm{N} 1-\mathrm{H} 2$ & 120.0 & $\mathrm{C} 4-\mathrm{C} 5-\mathrm{C} 6$ & $111.46(12)$ \\
\hline $\mathrm{H} 1-\mathrm{N} 1-\mathrm{H} 2$ & 120.0 & $\mathrm{C} 4-\mathrm{C} 5-\mathrm{H} 51$ & 109.3 \\
\hline $\mathrm{C} 9-\mathrm{C} 1-\mathrm{C} 6$ & $110.97(11)$ & $\mathrm{C} 6-\mathrm{C} 5-\mathrm{H} 51$ & 109.3 \\
\hline $\mathrm{C} 9-\mathrm{C} 1-\mathrm{C} 2$ & $110.51(10)$ & $\mathrm{C} 4-\mathrm{C} 5-\mathrm{H} 52$ & 109.3 \\
\hline $\mathrm{C} 6-\mathrm{C} 1-\mathrm{C} 2$ & $109.59(10)$ & $\mathrm{C} 6-\mathrm{C} 5-\mathrm{H} 52$ & 109.3 \\
\hline $\mathrm{C} 9-\mathrm{C} 1-\mathrm{C} 7$ & $106.94(10)$ & $\mathrm{H} 51-\mathrm{C} 5-\mathrm{H} 52$ & 108.0 \\
\hline $\mathrm{C} 6-\mathrm{C} 1-\mathrm{C} 7$ & $110.90(10)$ & $\mathrm{C} 5-\mathrm{C} 6-\mathrm{C} 1$ & $112.67(11)$ \\
\hline $\mathrm{C} 2-\mathrm{C} 1-\mathrm{C} 7$ & $107.86(10)$ & $\mathrm{C} 5-\mathrm{C} 6-\mathrm{H} 61$ & 109.1 \\
\hline $\mathrm{C} 3-\mathrm{C} 2-\mathrm{C} 1$ & $112.06(11)$ & $\mathrm{C} 1-\mathrm{C} 6-\mathrm{H} 61$ & 109.1 \\
\hline $\mathrm{C} 3-\mathrm{C} 2-\mathrm{H} 21$ & 109.2 & $\mathrm{C} 5-\mathrm{C} 6-\mathrm{H} 62$ & 109.1 \\
\hline $\mathrm{C} 1-\mathrm{C} 2-\mathrm{H} 21$ & 109.2 & $\mathrm{C} 1-\mathrm{C} 6-\mathrm{H} 62$ & 109.1 \\
\hline $\mathrm{C} 3-\mathrm{C} 2-\mathrm{H} 22$ & 109.2 & $\mathrm{H} 61-\mathrm{C} 6-\mathrm{H} 62$ & 107.8 \\
\hline $\mathrm{C} 1-\mathrm{C} 2-\mathrm{H} 22$ & 109.2 & $\mathrm{C} 8-\mathrm{C} 7-\mathrm{C} 1$ & $113.73(10)$ \\
\hline $\mathrm{H} 21-\mathrm{C} 2-\mathrm{H} 22$ & 107.9 & $\mathrm{C} 8-\mathrm{C} 7-\mathrm{H} 71$ & 108.8 \\
\hline $\mathrm{C} 2-\mathrm{C} 3-\mathrm{C} 4$ & $111.14(13)$ & $\mathrm{C} 1-\mathrm{C} 7-\mathrm{H} 71$ & 108.8 \\
\hline $\mathrm{C} 2-\mathrm{C} 3-\mathrm{H} 31$ & 109.4 & $\mathrm{C} 8-\mathrm{C} 7-\mathrm{H} 72$ & 108.8 \\
\hline $\mathrm{C} 4-\mathrm{C} 3-\mathrm{H} 31$ & 109.4 & $\mathrm{C} 1-\mathrm{C} 7-\mathrm{H} 72$ & 108.8 \\
\hline $\mathrm{C} 2-\mathrm{C} 3-\mathrm{H} 32$ & 109.4 & $\mathrm{H} 71-\mathrm{C} 7-\mathrm{H} 72$ & 107.7 \\
\hline $\mathrm{C} 4-\mathrm{C} 3-\mathrm{H} 32$ & 109.4 & $\mathrm{O} 1-\mathrm{C} 8-\mathrm{N} 1$ & $122.14(12)$ \\
\hline $\mathrm{H} 31-\mathrm{C} 3-\mathrm{H} 32$ & 108.0 & $\mathrm{O} 1-\mathrm{C} 8-\mathrm{C} 7$ & $120.10(12)$ \\
\hline $\mathrm{C} 5-\mathrm{C} 4-\mathrm{C} 3$ & $110.83(12)$ & $\mathrm{N} 1-\mathrm{C} 8-\mathrm{C} 7$ & $117.75(12)$ \\
\hline $\mathrm{C} 5-\mathrm{C} 4-\mathrm{H} 41$ & 109.5 & $\mathrm{O} 2-\mathrm{C} 9-\mathrm{O} 3$ & $123.19(12)$ \\
\hline $\mathrm{C} 3-\mathrm{C} 4-\mathrm{H} 41$ & 109.5 & $\mathrm{O} 2-\mathrm{C} 9-\mathrm{C} 1$ & $124.28(12)$ \\
\hline $\mathrm{C} 5-\mathrm{C} 4-\mathrm{H} 42$ & 109.5 & $\mathrm{O} 3-\mathrm{C} 9-\mathrm{C} 1$ & $112.48(11)$ \\
\hline $\mathrm{C} 9-\mathrm{C} 1-\mathrm{C} 2-\mathrm{C} 3$ & $-67.91(14)$ & $\mathrm{C} 6-\mathrm{C} 1-\mathrm{C} 7-\mathrm{C} 8$ & $-66.21(14)$ \\
\hline $\mathrm{C} 6-\mathrm{C} 1-\mathrm{C} 2-\mathrm{C} 3$ & $54.69(15)$ & $\mathrm{C} 2-\mathrm{C} 1-\mathrm{C} 7-\mathrm{C} 8$ & $173.78(11)$ \\
\hline $\mathrm{C} 7-\mathrm{C} 1-\mathrm{C} 2-\mathrm{C} 3$ & $175.52(12)$ & $\mathrm{C} 1-\mathrm{C} 7-\mathrm{C} 8-\mathrm{O} 1$ & $-70.50(15)$ \\
\hline $\mathrm{C} 1-\mathrm{C} 2-\mathrm{C} 3-\mathrm{C} 4$ & $-56.56(17)$ & $\mathrm{C} 1-\mathrm{C} 7-\mathrm{C} 8-\mathrm{N} 1$ & $108.96(13)$ \\
\hline
\end{tabular}


supporting information

$\begin{array}{llll}\mathrm{C} 2-\mathrm{C} 3-\mathrm{C} 4-\mathrm{C} 5 & 56.00(18) & \mathrm{C} 6-\mathrm{C} 1-\mathrm{C} 9-\mathrm{O} 2 & 13.30(17) \\ \mathrm{C} 3-\mathrm{C} 4-\mathrm{C} 5-\mathrm{C} 6 & -55.11(18) & \mathrm{C} 2-\mathrm{C} 1-\mathrm{C} 9-\mathrm{O} 2 & 135.09(13) \\ \mathrm{C} 4-\mathrm{C} 5-\mathrm{C} 6-\mathrm{C} 1 & 55.11(16) & \mathrm{C} 7-\mathrm{C} 1-\mathrm{C} 9-\mathrm{O} 2 & -107.76(14) \\ \mathrm{C} 9-\mathrm{C} 1-\mathrm{C} 6-\mathrm{C} 5 & 68.36(14) & \mathrm{C} 6-\mathrm{C} 1-\mathrm{C} 9-\mathrm{O} 3 & -169.07(11) \\ \mathrm{C} 2-\mathrm{C} 1-\mathrm{C} 6-\mathrm{C} 5 & -53.96(15) & \mathrm{C} 2-\mathrm{C} 1-\mathrm{C} 9-\mathrm{O} 3 & -47.28(14) \\ \mathrm{C} 7-\mathrm{C} 1-\mathrm{C} 6-\mathrm{C} 5 & -172.93(11) & \mathrm{C} 7-\mathrm{C} 1-\mathrm{C} 9-\mathrm{O} 3 & 69.87(13) \\ \mathrm{C} 9-\mathrm{C} 1-\mathrm{C} 7-\mathrm{C} 8 & 54.90(14) & & \end{array}$

Hydrogen-bond geometry $\left(\AA,{ }^{\circ}\right)$

\begin{tabular}{lllll}
\hline$D-\mathrm{H} \cdots A$ & $D-\mathrm{H}$ & $\mathrm{H} \cdots A$ & $D \cdots A$ & $D-\mathrm{H} \cdots A$ \\
\hline $\mathrm{O} 3-\mathrm{H} 3 \cdots \mathrm{O} 1^{\mathrm{i}}$ & $0.963(18)$ & $1.640(19)$ & $2.5829(13)$ & $165(2)$ \\
$\mathrm{N} 1-\mathrm{H} 1 \cdots \mathrm{O} 1^{\mathrm{ii}}$ & 0.88 & 2.21 & $3.0680(15)$ & 164 \\
$\mathrm{~N} 1-\mathrm{H} 2 \cdots \mathrm{O} 2^{\mathrm{iii}}$ & 0.88 & 2.12 & $2.9635(15)$ & 162
\end{tabular}

Symmetry codes: (i) $-x, y-1 / 2,-z+1 / 2$; (ii) $-x, y+1 / 2,-z+1 / 2$; (iii) $x,-y+1 / 2, z-1 / 2$. 